\title{
A Short Proof of a Gauss Problem *
}

\author{
H. de Fraysseix and P. Ossona de Mendez \\ CNRS UMR 0017, EHESS, 54 Boulevard Raspail, 75006, Paris, France. \\ email : hf@ehess.fr, pom@ehess.fr
}

\begin{abstract}
The traversal of a self crossing closed plane curve, with points of multiplicity at most two, defines a double occurrence sequence.

C.F. Gauss conjectured [2] that such sequences could be characterized by their interlacement properties. This conjecture was proved by P. Rosenstiehl in 1976 [15]. We shall give here a simple self-contained proof of his characterization. This new proof relies on the $\mathrm{D}$-switch operation.
\end{abstract}

\section{Introduction}

We first recall and introduce some definitions and notations concerning geometric properties of closed plane curves. For related topics, we refer the reader to the bibliography. P. Rosenstiehl exposed recently a new proof of this theorem, based on patches, that will soon be published.

A parameterized curve $C$ is a continuous mapping $C:[0,1] \rightarrow \mathbb{R}^{2}$, such that $C(0)=$ $C(1)$ and such that the underlying curve $C([0,1])$ of $C$ has a finite number of multiple points, which all have multiplicity two. Let $P(C)$ denote the set of the points of multiplicity two. To any point $p \in P(C)$, we associate the two parameter values $t_{p}^{\prime}$ and $t_{p}^{\prime \prime}$, such that $t_{p}^{\prime}<t_{p}^{\prime \prime}$ and $C\left(t_{p}^{\prime}\right)=C\left(t_{p}^{\prime \prime}\right)=p$. A point $p \in P(C)$ is a crossing point if any local deformation of $C$ in a neighborhood of $t_{p}^{\prime}$ preserves the existence of a double point. Otherwise, $p$ is a touching point. A touch curve (resp. a cross curve) is a parameterized curve with touching points (resp. crossing points) only.

There are two different types of touching points, depending on the local behavior of the parameterized curve :

Type 1

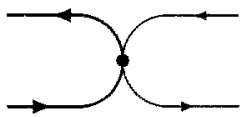

Type 2

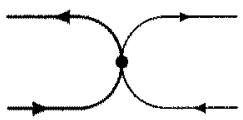

Remark. All the touch points of a touch curve are of type 1.

The sequence of the points of $P(C)$ encountered while the parameter $t$ goes from 0 to 1 (excluded) is the traversal sequence of $C$ and is denoted by $S(C)$.

In the following, sequences are understood to have two occurrences of each symbol and to be defined up to reversal and cyclic permutation. Given a sequence $S$, two symbols $p, q$ are interlaced in $S$ if exactly one occurrence of $q$ appears in $S$ between the two occurrences of $p$. We shall denote by $A(S)$ the interlacement graph of $S$ defined by the interlacement relation in $S$.

A sequence $S$ is realized by a parameterized curve $C$ if $S$ is the traversal sequence of $C$. A sequence is touch realizable (resp. cross realizable) if it can be realized by a touch curve (resp. a cross curve).

\footnotetext{
* This work was partially supported by the Esprit LTR Project no 20244-ALCOM IT.
} 


\section{Switches and D-switches}

Let us introduce the switch operation $[4,8]$ : Given a point $p$ of $P(C)$, the curve $C^{\prime}=C$ o $p$ is defined by :

$$
C^{\prime}(t)= \begin{cases}C(t), & \text { if } t \notin\left[t_{p}^{\prime}, t_{p}^{\prime \prime}\right] \\ C\left(t_{p}^{\prime}+t_{p}^{\prime \prime}-t\right), & \text { if } t \in\left[t_{p}^{\prime}, t_{p}^{\prime \prime}\right]\end{cases}
$$

This curves as the same touching and crossing points as $C$, with the possible exception of $p$. The traversal sequence of $C^{\prime}$ is obtained from the one of $C$ by inverting the order of the points encountered between the two occurrences of $p$. We shall say that the points that are interlaced with $p$ have been inverted. The switch operation on $S$ will be denoted by $S \circ p$, so that : $S(C \circ p)=S(C) \circ p$. Let us remark that these switch operations are involutions: $C \circ p \circ p=C$ and $S \circ p \circ p=S$.

Remark. A switch at a point $p$ of a parameterized curve transforms $p$ in the following way :

- touching point of type $1 \leftrightarrow$ crossing point,

- touching point of type $2 \leftrightarrow$ touching point of type 2 .
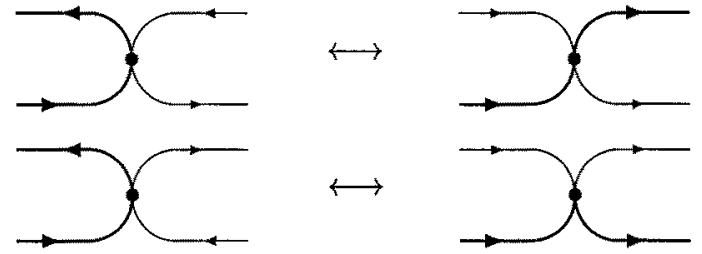

Remark. If $q$ is a touching point of $C$ different from $p$, then $q$ is a touching point with a type different in $C$ and $C \circ p$ if and only $p$ and $q$ are interlaced (that is if $q$ has been inverted by the switch at $p$ ).

The switch of a point $p$ in a sequence $S$ induces a local complementation of $p$ in the interlacement graph $A(S)$ : two symbols $a, b$ are adjacent in $\Lambda(S \circ p)$ if and only if

- $a$ or $b$ is not adjacent to $p$ in $A(S)$ and $(a, b)$ is an edge of $A(S)$, or

- $a$ and $b$ are both adjacent to $p$ in $A(S)$ and $(a, b)$ is not an edge of $A(S)$.

For sake of simplicity, the local complementation of $p$ in $\Lambda(S)$ will be denoted by $\Lambda(S) \circ p$, so that $A(S \circ p)=\Lambda(S) \circ p$.

Let $S$ be a sequence, a $D$-switch at $p$ consists in a switching at $p$ and in the adding of two occurrences of a new symbol $p^{\prime}$ (called twin of $p$ ), one just after the first occurrence of $p$ and one just before the second occurrence of $p$.

$$
S=(\alpha p \beta p \gamma) \mapsto S \otimes p=\left(\alpha p p^{\prime} \beta^{-1} p^{\prime} p \gamma\right)
$$

A D-switch of $p$ in $S$ corresponds in $\Lambda(S)$ to a local complementation of $p$ and the addition of a new vertex having the same neighbors as $p$. This graph operation will be similarly denoted by ' $\oplus$ ', so that $\Lambda(S) \oplus p=\Lambda(S \oplus p)$.

Remark. The sequence obtained from $S \oplus p \oplus p$ by deleting the two twins of $p$ is equal to $S$. 


\section{On realizable sequences}

We first state two propositions proved by Dehn, which follow from the remarks of the preceding sections.

Proposition 1. Consider a cross curve $C$ and any given order $\left(p_{1}, \ldots, p_{n}\right)$ of the points of $C$. Then, the parameterized curve $C \circ p_{1} \circ \ldots \circ p_{n}$ obtained from $C$ by switching successively the $p_{i}$ is a touch curve.

The converse of this proposition is not true (e.g. the sequence ( $a b a b)$ is not cross realizable).

Remarks. Let us note by $S \stackrel{\circ}{\rightarrow} S^{\prime}$ the existence of an order $\left(p_{1}, \ldots, p_{n}\right)$ of the symbols of $S$, such that $S^{\prime}=S \circ p_{1} \circ \ldots \circ p_{n}$.

- A cross realizable sequence does not determine the cross curve itself up to an homeomorphism: actually, a cross realizable sequence $S$ can be proved to be realized by $2^{c(A(S))-1}$, where $c(\Lambda(S))$ is the number of connected components of the interlacement graph of $S$.

- One may find a cross realizable sequence $S_{1}$, a non cross realizable sequence $S_{2}$ and a touch realizable sequence $S_{T}$, such that $S_{1} \stackrel{\circ}{\longrightarrow} S_{T}$ and $S_{2} \stackrel{\circ}{\longrightarrow} S_{T}$. Actually, $S_{1}$ and $S_{2}$ may be proved to have different interlacement graph (using the main theorem).

- Two different cross realizable sequences $S_{1}$ and $S_{2}$ may have the same interlacement graph (e.g. the sequences (abcaefdcbe $f d$ ) and (acbaefdbce $f d)$ ). However, no sequence $S_{T}$ satisfies $S_{1} \stackrel{\circ}{\longrightarrow} S_{T}$ and $S_{2} \stackrel{\circ}{\longrightarrow} S_{T}$.

Proposition 2. A sequence $S$ is touch realizable if and only if its interlacement graph $\Lambda(S)$ is bipartite.

Proof. The figure bellow shows how a touch curve may be transformed into a bipartite chord diagram with the same interlacement (and conversely).
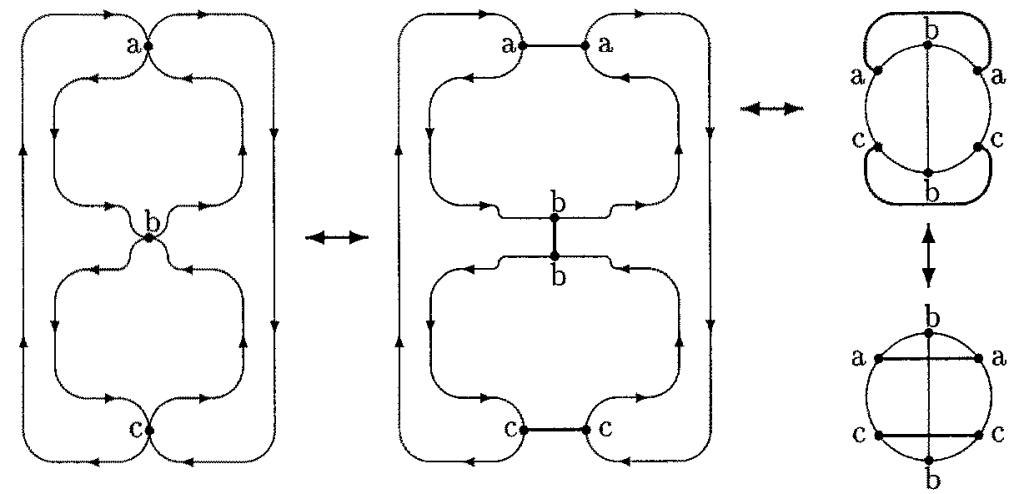

Theorem 3, Let $S$ be a sequence, and let $\left(p_{1}, \ldots, p_{n}\right)$ be any order on its symbols. Then, $S$ is cross realizable if and only if the sequence $S_{n}=S \oplus p_{1} \otimes \ldots \oplus p_{n}$ obtained by successively $D$-switching the $p_{i}$ has a bipartite interlacement graph.

Proof. - Assume $S$ is realized by a cross curve $C$. As a D-switch of a crossing point of a parameterized curve gives rise to two touching points (that will never become crossing points again), the curve $C$ is iteratively transformed into a touch curve $C_{n}$. The traversal sequence $S_{n}$ of $C_{n}$ has hence a bipartite interlacement graph. 
- Conversely, assume that $S_{n}$ has a bipartite interlacement graph.

Let $S_{i}=S \otimes p_{1} \oplus \ldots \oplus p_{i}$ denote the sequence obtained after the first $i \mathrm{D}$-switches, we shall inductively construct (for $i$ going from $n$ to 0 ) a parameterized curve $C_{i}$, that realizes $S_{i}$, and such that the crossings of $C_{i}$ are the $p_{j}$, with $j>i$. Then, the parameterized curve $C_{0}$ will be a cross curve realizing $S$.

As $\Lambda\left(S_{n}\right)$ is bipartite, there exists a touching curve $C_{n}$ whose traversal sequence is $S_{n}$. If $p_{i}$ is of type 1 , the suppression of $p_{i}^{\prime}$ and the switch of $p_{i}$ transforms $p_{i}$ into a crossing point and gives rise to a parameterized curve $C_{i-1}$, having $p_{i}, \ldots, p_{n}$ as crossing points and $S_{i-1}$ as traversal sequence. The recursion is then complete if only this case may occur.

So, we shall prove that $p_{i}$ is always of type 1 in $C_{i}$, that is that $p_{i}$ has been inverted an even number of times during the D-switch at $p_{i}, \ldots, p_{n}$ : The symbol $p_{i}$ and its twin $p_{i}^{\prime}$ are not interlaced in $S_{i}$, they are alternatively interlaced and not interlaced after each further inversion and, if $p_{i}$ has been last inverted by a switch at $p_{j}, p_{i}$ (resp. $p_{i}^{\prime}$ ) and $p_{j}$ are interlaced in $S_{n}$. As $\Lambda\left(S_{n}\right)$ is bipartite, $p_{i}$ and $p_{i}^{\prime}$ are not interlaced in $S_{n}$ (else $p_{i}, p_{i}^{\prime}, p_{j}$ would define a triangle of $\left.\Lambda\left(S_{n}\right)\right)$. Hence, the symbol $p_{i}$ has been inverted an even number of times.

Remark. A cross curve realizing the sequence $S$ could be geometrically derived from a touch curve realizing the sequence $S^{\prime}$ obtained from $S_{n}$ by suppressing all twined letters by transforming each touching point into a crossing point.

\section{Proof of Rosenstiehl's Theorem}

Definition 4. Let $G$ be a graph and let $(A, B)$ be a bipartition of its vertex set.

The property $P(G ; A, B)$ is satisfied by a pair $\{u, v\}$ of vertices of $G$ whenever the following equivalence holds :

- the vertices $u$ and $v$ have an odd number of common neighbors,

- the vertices $u$ and $v$ are adjacent and belong to the same class $(A$ or $B$ ).

Lemma 5. Let $G$ be a graph with a vertex bipartition $A, B$ and let $p$ be a vertex of $G$. Let $G^{\prime}=G \otimes p$ and let $A^{\prime}, B^{\prime}$ be the vertex bipartition of $G^{\prime}$ defined by: $A^{\prime}=A+N(p), B^{\prime}=$ $B+N(p)$ and assigning $p^{\prime}$ to the class of $p$.

If $G$ is eulerian and any pair $\{u, v\}$ of vertices of $G$ satisfies $P(G ; A, B)$, then $G^{\prime}$ is eulerian and any pair $u, v$ of vertices of $G^{\prime}$ satisfies $P\left(G^{\prime} ; A^{\prime}, B^{\prime}\right)$.

Proof. We have the following relationship between the neighborhood $N_{G^{\prime}}$ in $G^{\prime}$ and the neighborhood $N_{G}$ in $G$ :

- $N_{G^{\prime}}(u)=N_{G}(u)$, if $u$ is not adjacent to $p$ (in $G$ or equivalently in $G^{\prime}$ ),

$-N_{G^{\prime}}\left(p^{\prime}\right)=N_{G^{\prime}}(p)=N_{G}(p)$

$-N_{G^{\prime}}(u)=N_{G}(u)+u+N_{G}(p)+p^{\prime}$, if $u$ is adjacent to $p$.

In order to prove that $G^{\prime}$ is eulerian, we only have to check that the neighbors of $p$ have an even degree : the parity of $N_{G^{\prime}}(u)=N_{G}(u)+u+N_{G}(p)+p^{\prime}$ is the sum of the parities of $N_{G}(u),\{u\}, N_{G}(p)$ and $\{p\}$ and hence is even.

Now, we shall prove that any pair $\{u, v\}$ of vertices of $G^{\prime}$ satisfies $P\left(G^{\prime} ; A^{\prime}, B^{\prime}\right)$. If $u$ or $v$ is $p^{\prime}$, we shall replace it by $p$ as $p$ and $p^{\prime}$ have the same neighbors, are not adjacent and belong to the same class $\left(A^{\prime}, B^{\prime}\right)$. 
If two vertices $u, v$ are not adjacent or equal to $p$, then their adjacencies, their class and their number of common neighbors are the same in $G$ and $G^{\prime}$. Thus, the pair $u, v$ satisfies $P\left(G^{\prime} ; A^{\prime}, B^{\prime}\right)$.

If $u$ is adjacent to $p$ and $v$ is not adjacent or equal to $p$, then

$$
\begin{aligned}
<N_{G^{\prime}}(u), N_{G^{\prime}}(v)> & =\left\langle N_{G}(u)+u+N_{G}(p)+p^{\prime}, N_{G}(v)>\right. \\
& =\left\langle N_{G}(u), N_{G}(v)\right\rangle+1
\end{aligned}
$$

As $u$ and $v$ belong to the same class $\left(A^{\prime}, B^{\prime}\right)$ if and only if they do not belong to the same class $(A, B)$ and as they are adjacent, the pair $u, v$ satisfies $P\left(G^{\prime} ; A^{\prime}, B^{\prime}\right)$.

If $u$ and $v$ are both adjacent to $p$, then

$$
\begin{aligned}
<N_{G^{\prime}}(u), N_{G^{\prime}}(v)> & =<N_{G}(u)+u+N_{G}(p)+p^{\prime}, N_{G}(u)+v+N_{G}(p)+p^{\prime}> \\
& =<N_{G}(u), N_{G}(v)>+<N(u), N(p)>+<N(v), N(p)>+1
\end{aligned}
$$

As $u$ is adjacent to $p,\langle N(u), N(p)\rangle=1$ if and only if $u$ and $p$ belong to the same class $(A, B)$. So, $\langle N(u), N(p)\rangle+\langle N(v), N(p)\rangle+1=1$ if and only if $u$ and $v$ belong to the same class $(A, B)$. As $\left\langle N_{G}(u), N_{G}(v)>=1\right.$ if and only if $u$ and $v$ are adjacent in $G$ and belong to the same class $(A, B),\left\langle N_{G^{\prime}}(u), N_{G^{\prime}}(v)\right\rangle=1$ if and only if $u$ and $v$ are not adjacent in $G$ and belong to the same class $(A, B)$, that is, if and only if they are adjacent in $G^{\prime}$ and belong to the same class $\left(A^{\prime}, B^{\prime}\right)$. Thus, the pair $u, v$ satisfies $P\left(G^{\prime} ; A^{\prime}, B^{\prime}\right)$.

Lemma 6. Let $G$ be a graph with a vertex bipartition $A, B$ and let $p$ be a vertex of $G$. Let $G^{\prime}=G \oplus p$ and let $A^{\prime}, B^{\prime}$ be the vertex bipartition of $G^{\prime}$ defined by: $A^{\prime}=A+N(p), B^{\prime}=$ $B+N(p)$ and assigning $p^{\prime}$ to the class of $p$. If $G^{\prime}$ is eulerian and any pair $\{u, v\}$ of vertices of $G^{\prime}$ satisfies $P\left(G^{\prime} ; A^{\prime}, B^{\prime}\right)$, then $G$ is eulerian and any pair $\{u, v\}$ of vertices of $G$ satisfies $P(G ; A, B)$.

Proof. By Lemma 5, $G^{\prime \prime}=G^{\prime} \oplus p$ has the requested property and this property is still satisfied when deleting the two twins of $p$.

Theorem 7 (RosenstienL)[15]. A sequence $S$ is cross realizable if and only if its interlacement graph $\Lambda(S)$ satisfies:

- the graph is eulerian,

- for any non-edge $\left(p, p^{\prime}\right)$ of the graph, $N(p) \cap N\left(p^{\prime}\right)$ is even,

- the set of the of the edges $\left(p, p^{\prime}\right)$ of the graph such that $N(p) \cap N\left(p^{\prime}\right)$ is even is a cocycle of the graph.

Proof. The theorem may be restated as follows: A sequence $S$ is cross realizable if and only if its interlacement graph $\Lambda(S)$ is eulerian and if there exists a bipartition $A, B$ of the vertex set of $A(S)$ such that any pair $u, v$ of vertices of $A(S)$ satisfies $P(A(S) ; A, B)$.

Consider any sequence $S_{n}=S \otimes p_{1} \otimes \ldots \oplus p_{n}$ obtained by successively D-switching the symbols of $S$. According to Lemma $5, A\left(S_{n}\right)$ is eulerian and has a bipartition $A^{\prime}, B^{\prime}$ such that any pair of vertices of $A\left(S_{n}\right)$ satisfies $P\left(\Lambda\left(S_{n}\right) ; A^{\prime}, B^{\prime}\right)$. As all the symbols have been twined and as $p$ and its twin $p^{\prime}$ have the same neighbors, any two vertices of $A\left(S_{n}\right)$ have an even number of common vertices. According to property $P\left(A\left(S_{n}\right) ; A^{\prime}, B^{\prime}\right)$, the graph $A\left(S_{n}\right)$ is bipartite. Then, from Theorem $3, S$ is cross realizable.

Conversely, if $S$ is cross realizable, any sequence of D-switches gives rise to a sequence $S^{t}$ having a bipartite interlacement graph. This graph is eulerian (due to the doubling of each symbol) and a bipartition $A, B$ induced by a bicoloration, is such that each pair of vertices satisfies $P\left(A\left(S^{\prime}\right) ; A, B\right)$. The theorem then follows from Lemma 6 . 


\section{Matroidal Interpretation}

As we wanted to give a short self-contained proof, we did not introduce the usual concepts of binary matroids. In such a context, a proof could be done, relying on the following properties: The graphs which satisfy the conditions given for $A(S)$ in Rosenstiehl's characterization are exactly the principal interlacement graph of some binary matroid $M$ [14]. Any local complementation of the vertices of such a graph gives rise to a bipartite graph, which is the fundamental interlacement graph of $M$ with respect to some base $B$ of $M[3]$. The further condition that a principal interlacement graph is an interlacement graph (that is a circle graph) implies that the matroid $M$ is planar and then, the principal interlacement graph corresponds to the interlacement of a left-right path of a planar realization of $M[3]$.

\section{References}

1. A. Bouchet. Caractérisation des symboles croisés de genre nul. C.R. Acad. Sci., 274:724-727, 1972. (Paris).

2. H. de Fraysseix. Sur la représentation d'une suite à triples et à doubles occurrences par la suite des points d'intersection d'une courbe fermée du plan. In Problèmes combinatoires et théorie des graphes, volume 260 of Colloques internationaux C.N.R.S., pages 161-165. C.N.R.S., 1976.

3. H. de Fraysseix. Local complementation and interlacement graphs. Discrete Mathematics, 33:29-35, 1981.

4. M. Dehn. Über kombinatorische topologie. Acta Math., 67:123-168, 1936. (Sweden).

5. H. Fleischner. Cycle decompositions, 2-coverings, removable cycles, and the four-colordisease. Progress in Graph Theory, pages 233-246, 1984.

6. G.K. Francis. Null genus realizability criterion for abstract intersection sequences. $J$. Combinatorial Theory, 7:331-341, 1969.

7. C.F. Gauss. Werke, pages 272 and 282-286. Teubner Leipzig, 1900.

8. A. Kotzig. Eulerian lines in finite 4-valent graphs and their transformations. In Proceedings of the Colloquium held at Tihany, Hungary, pages 219-230, 1969.

9. L. Lovász and M.L. Marx. A forbidden subgraph characterization of gauss codes. Bull. Am. Math. Soc., 82:121-122, 1976.

10. M.L. Marx. The gauss realizability problem. Trans Am. Math. Soc., 134:610-613, 1972.

11. J.V.Sz. Nagy. Über ein topologisches problem von gauss. Maht. Z., 26:579-592,1927. (Paris).

12. R.C. Read and P. Rosenstiehl. On the gauss crossing problem. In Colloquia Mathematica Societatis János Bolyai, pages 843-875, 1976 . (Hungary).

13. R.C. Read and P. Rosenstiehl. On the principal edge tripartition of a graph. Annals of Discrete Maths, 3:195-226, 1978.

14. P. Rosenstiehl. Les graphes d'entrelacement d'un graphe. In Problèmes combinatoires et théorie des graphes, volume 260 of Colloques internationaux C.N.R.S., pages 359-362. C.N.R.S., 1976.

15. P. Rosenstiehl. Solution algébrique du problème de gauss sur la permutation des points d'intersection d'une ou plusieurs courbes fermées du plan. C.R. Acad. Sci., 283 (A):551-553, 1976. (Paris).

16. P. Rosenstiehl. A geometric proof of a Gauss crossingg problem. (to appear).

17. P. Rosenstiehl and R.E. Tarjan. Gauss codes, planar hamiltonian graphs, and stack-sortable permutations. Jour. of Algorithms, 5:375-390, 1984.

18. H. Shank. The theory of left-right paths. In Combinatorial Math., volume III of Lecture Notes in Math., pages 42-54. Springer, 1975.

19. W.T. Tutte. On unicursal paths in a network of degree 4. Amer. Math. Monthly, 4:233-237, 1941. 